calculated for each component for each patient (table 1). Patient Reported Outcomes (PROs) regarding pain (visual analogue scale), numbers of attacks within the last 3 months and physical function (Health Assessment Questionnaire) were obtained, as were $\mathrm{C}$-reactive protein (CRP), p-urate and clinical joint examination All examinations were repeated after $3(n=29)$ and 6 months $(n=15$, follow-up still ongoing) and changes in scores were evaluated using Wilcoxon-Pratt signedrank test.

Results: 29 patients (28 males, 1 female), mean age of $68(39-89)$ years were included. US showed a numerical, but statistically non-significant $(p=0.13)$, decline in DC count from baseline to 3 months' follow up, while at 6 months a statistically significant decline was observed $(p=0.033)$. The tophus count decreased non-significantly at both 3 and 6 months' follow up, whereas the aggregate and erosion counts by large were unchanged. GS synovitis showed a statistically nonsignificant decrease at follow ups, whereas CD synovitis and SC oedema counts declined significantly at 3 months' follow up ( $p=0.033$ and 0.044 , respectively).

$\mathrm{P}$-urate levels decreased statistically significant from baseline to both 3 and 6 months' follow-up (both p-values $<0.001$ ), as did clinical markers such as CRP, joint evaluation, pain and attack frequency.

Abstract OP0155 - Table 1 Course of US, biochemical and clinical variables during urate lowering therapy

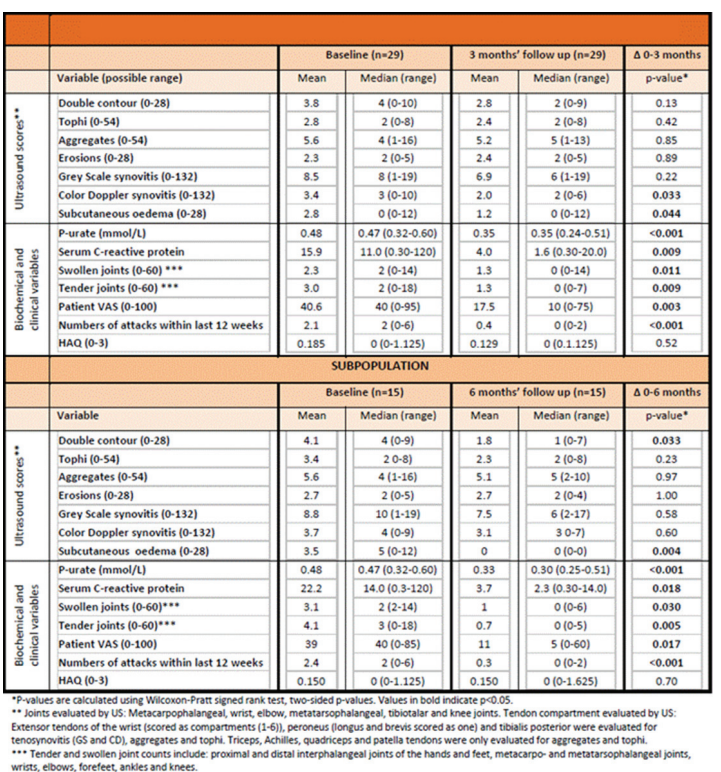

Conclusions: Of the four OMERACT US elementary gout lesions only DC count showed a statistically significant decrease as a response to 6 months of urate lowering therapy. The number of tophi had decreased at both 3 and 6 months' follow up, but not statistically significant. Aggregates and erosions count did not markedly respond to the 6 month treatment.

The study indicates that US assessing the OMERACT elementary lesions, particularly DC, is a feasible tool for monitoring gout lesions. However, a follow-up of at least 6 months may be needed to detect change of crystal deposits, as reflected by $\mathrm{DC}$, and presumably an even longer follow-up period is needed to evaluate more massive deposits as tophi.

Disclosure of Interest: None declared

DOI: 10.1136/annrheumdis-2018-eular.2209

\section{OP0156 SIMPLE ASSESSMENT OF CONVENTIONAL 18F-FDG PET/CT ACCURATELY DIAGNOSES CRANIAL ARTERITIS IN GLUCOCORTICOID-NAÏVE GCA PATIENTS: A CASE-CONTROL STUDY}

B.D Nielsen ${ }^{1,2}$, I.T. Hansen ${ }^{3,4}$, A. Haraldsen ${ }^{5}$, S. Kramer ${ }^{5}$, K.K. Keller ${ }^{1}$, P. Therkildsen ${ }^{1}$, E.-M. Hauge ${ }^{1}$, L.C. Gormsen ${ }^{1} .{ }^{1}$ Rheumathology; ${ }^{2}$ Clinical medicine; ${ }^{3}$ Rheumatology, Aarhus University Hospital; ${ }^{4}$ Clinical medicine, Aarhus University;

${ }^{5}$ Nuclear Medicine and PET, Aarhus University Hospital, Aarhus, Denmark

Background: Although older studies argue that fluorine-18-fluorodeoxyglucose (FDG) positron emissions tomography (PET)/CT cannot demonstrate inflammation in cranial arteries the spatial resolution of modern PET systems have greatly improved allowing for more precise diagnostics of small structures. FDG PET/CT is widely used to diagnose large-vessel (LV) giant cell arteritis (GCA). Recognising FDG uptake in cranial arteries potentially adds to FDG PET/CTs diagnostic accuracy for GCA.

Objectives: To evaluate the diagnostic accuracy of conventional FDG PET/CT of the cranial arteries in GCA.

Methods: In a cohort of consecutively included glucocorticoid-naïve patients sus pected of new-onset GCA, patients full-filling 1990 ACR criteria for GCA were identified. Conventional FDG PET/CT and clinical assessment was performed before treatment. Controls were age- and sex-matched patients with malignant melanoma (MM) who had a metastatic-disease-free follow-up FDG PET/CT $\geq 6$ months after MM resection.

All PET images were evenly cropped to include only head and neck. Images were randomly assessed by 2 nuclear medicine physicians (10 years experience) blinded to clinical symptoms and findings. Training included review of 5 GCA-PET examinations (not part of cohort). Temporal (TA), maxillary (MA) and vertebra (VA) arteries were visually scored bilaterally. Arterial FDG uptake above surrounding tissue was considered indicative of inflammation and graded low or high. If disagreement between readers occurred, final score was settled by an expert nuclear medicine physician.

Student t test was used for quantitative data. Inter-reader agreement was evaluated by Cohens weighted kappa (disagreement on diagnosis weighted 0 , disagreement on FDG uptake intensity weighted 0.2).

Results: A total of 44 patients and 44 controls were identified. In both case and control group, the mean age was 69 years $(p=0.45)$ and 25/44 were women. Large-vessel involvement was seen in 39/42 patients, and 35/42 were temporal artery biopsy positive. GCA patients' median global assessment of disease activity was 8 (IQR: $5-10)$ and median CRP was $70(95 \% \mathrm{Cl}: 58 ; 85) \mathrm{mg} / \mathrm{L}$.

Considering only FDG uptake in TA and/or MA, diagnostic sensitivity and specificity was $66 \%(95 \% \mathrm{Cl}: 50 \%-80 \%)$ and $100 \%$ (95\% Cl: 92\%-100\%). Including VA, sensitivity increased to $86 \%(95 \% \mathrm{Cl}: 73 \%-95 \%)$ and specificity remained high, $98 \%$ (95\% Cl: $88 \%$ to $100 \%$ ). Cohens weighted kappa was 0.82 (agreement $93 \%$, $\mathrm{p}=0.000$ ) in a per segment analysis and kappa was 0.84 (agreement $92 \%$, $p=0.000)$ in diagnosis.

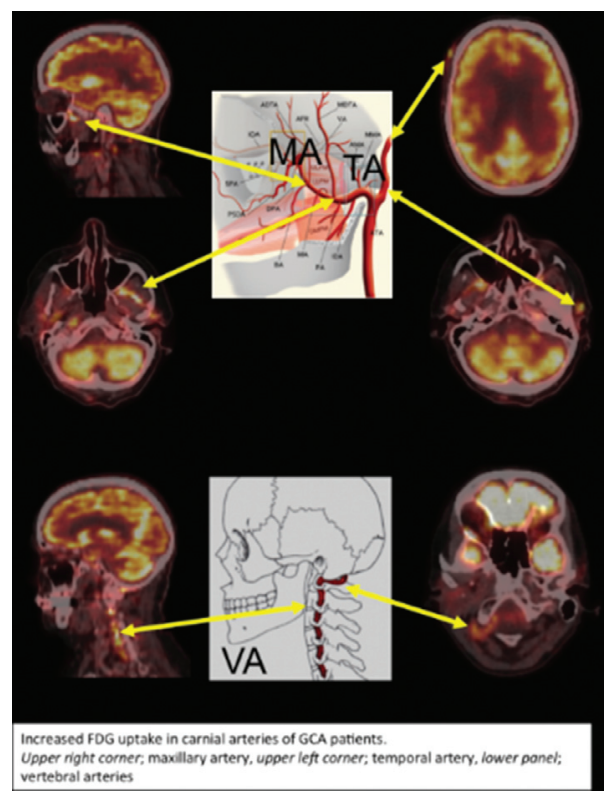

Absatrct OP0156 - Figure 1 FDG PET/CT of cranial arteries in patients with giant cell arteritis

Conclusions: Inter-reader agreement on FDG uptake in cranial arteries is almost perfect, and cranial arteritis in glucocorticoid-naïve GCA patients can be readily and accurately diagnosed by conventional FDG PET/CT. The high diagnostic specificity suggests that TAB can be avoided in patients with FDG uptake in cranial arteries. Moreover, FDG PET/CT performed in patients with suspected vasculitis should always include head and neck.

Disclosure of Interest: None declared

DOI: 10.1136/annrheumdis-2018-eular.1338 